\title{
因HAD
}

DOI: http://doi.org/10.22585/hospdomic.v2i2.45

\section{Influencia de los suplementos nutricionales en la evolución de las heridas quirúrgicas abdominales complicadas de pacientes en hospitalización a domicilio}

\section{Influence of nutritional supplements on the evolution of complicated abdominal surgical wounds of a group of patients in home hospitalization}

Carmina Wanden-Berghe', Nieves Santacruz Carmona', Catalina Cases Alarcón', Lourdes Granell', Teresa Gonzalez Valls', Marian Miralles', Arantxa García'

1. Hospital General Universitario de Alicante. Instituto de Investigación Sanitaria y Biomédica de Alicante ISABIAL-FISABIO.

Correspondencia/Correspondence

Carmina Wanden-Berghe Lozano

Hospital General Universitario de Alicante, Pintor Baeza s/n, Alicante 03010

carminaw@telefonica.net

Recibido/Received

05.03.2018

Aceptado/Accepted

07.03 .2018
Conflicto de Intereses/Competing interest Los autoras, en su totalidad declaran que no hay ningún posible conflicto de interés

Financiación/Funding

No se ha recibido ninguna beca o apoyo económico para la investigación de la que deriva el artículo
Agradecimientos/Acknowledgments
Este trabajo fue presentado previamente a su publicación en forma de póster en el 31 congreso de la Sociedad Española de Nutrición Parenteral y Enteral, recibiendo la consideración del premio a la mejor comunicación realizada con inmunonutrientes.

CÓMO CITAR ESTE TRABAJO | HOW TO CITE THIS PAPER

Wanden-Berghe C, Santacruz Carmona N, Cases Alarcón C, Granell L, Gonzalez Valls T, Miralles M, García A. Influencia de los suplementos nutricionales en la evolución de las heridas quirúrgicas abdominales complicadas de pacientes en hospitalización a domicilio. Hosp Domic. 2018;2(2):49-53 


\section{RESUMEN}

Objetivo: Evaluar el impacto de los suplementos nutricionales en pacientes ingresados en la Unidad de Hospitalización a Domicilio con heridas quirúrgicas abdominales complicadas.

Material y Métodos: Estudio retrospectivo de 67 pacientes que a su ingreso en la Unidad de Hospitalización a Domicilio presentaban heridas quirúrgicas abdominales complicadas. Se estudió el tipo y cantidad de suplemento recibido, Índice de Masa Corporal (IMC), albumina, tiempo y ciclos de antibioterapia.

Resultados: La edad media de los 67 pacientes fue de $69,2 \pm 5,6$. Todos los pacientes presentaban heridas abdominales abiertas y el 53,7\% presentaban infección con antibioterapia intravenosa. Todos los pacientes recibieron algún tipo de suplemento nutricional (estándar 62,7\%; inmunomodulador (Atempero®) 25,4\% y otros $11,9 \%$ ). Al mes de seguimiento el valor medio de albumina se había incrementado en todos los pacientes $p=0,032$ aunque la mayor diferencia la presentaron los pacientes que recibieron nutrición inmunomoduladora $p=0,001$. La estancia media fue de 84,05 $\pm 17,48$ días hasta el alta, en el grupo del suplemento inmunomodulador fue de $77,18 \pm 13,72$ días $(p=0,001)$.

Conclusiones: Los datos apoyan un beneficio de la suplementación oral en este tipo de pacientes mejorando su estado nutricional. El grupo que se suplementó con Atempero®, destacó tanto en la recuperación de los niveles de albumina como en una mejor evolución, lo que se traduce en un alta significativamente más precoz.

Palabras clave: Soporte nutricional; Herida quirúrgica; Complicación postquirúrgica

\section{ABSTRACT}

Objective: To evaluate the impact of nutritional supplements in patients admitted to the Home Hospitalization Unit with complicated abdominal surgical wounds.

Material and methods: We have performed a retrospective study of 67 patients which presented complicated abdominal surgical wounds upon admission to the Home Hospitalization Unit. We have studied the type and amount of the supplement received, the body mass index (BMI), the albumin, and the time and cycles of antibiotic therapy.

Results: The median age of the 67 patients was $69.2 \pm$ 5.6. All patients had open abdominal wounds and $53.7 \%$ had infection with intravenous antibiotics. All patients received some type of nutritional supplement (62.7\% standard, $25.4 \%$ immunomodulatory (Atempero®), 11.9\% others). After one month of follow-up, the albumin mean value had increased in all patients $p$ $=0.032$, although the greatest difference was in patients who received immunomodulatory nutrition $p=0.001$. The median follow-up was $84.05 \pm 17.48$ days until discharge. In the immunomodulatory supplement group it was $77.18 \pm$ 13.72 days $(p=0.001)$.

Conclusions: The data support a benefit of oral supplementation in this type of patients improving their nutritional status. The group that was supplemented with Atempero®, stood out both in the recovery of albumin levels and in a better evolution, which translates into a significantly earlier discharge.

Keywords: Nutritional support; Surgical wounds; Post-surgical complication. 


\section{INTRODUCCIÓN}

El concepto de nutrientes como «sustancias asimilables que contenidas en los alimentos, permite al organismo obtener energía, construir y reparar tejidos, y regular los procesos metabólicos», se ha complementado con el de inmunonutrientes, que añade, además de las propiedades de un nutriente convencional, «la capacidad de mejorar el sistema inmunitario» (1).

En los últimos años, las fórmulas nutricionales estándar se han modificado mediante la adición de arginina, ácidos grasos omega-3, glutamina, nucleótidos y otros componentes, que pueden aumentar la respuesta inmune al modular el proceso inflamatorio o mejorar la síntesis de proteínas después de la cirugía. Los efectos potenciales de estos inmunonutrientes incluyen la reducción de complicaciones infecciosas y otras complicaciones postoperatorias. Los suplementos nutricionales enriquecidos con inmunonutrientes se han ido introducido en la práctica clínica.

La inmunosupresión causada por el estrés quirúrgico es uno de los factores más importantes en la evolución de los pacientes sometidos a cirugía. Las infecciones se mantienen entre los principales problemas que siguen a la cirugía colorrectal (2). Es difícil anticipar cuándo ocurren tales complicaciones porque sus causas son variadas. La suplementación nutricional en los pacientes quirúrgicos con fórmulas de inmunonutrientes, puede ser una aportación importante al curso del postoperatorio. Se ha comprobado en un ensayo clínico que su aporte en el preoperatorio a pacientes normonutridos sometidos a cirugía colorrectal reduce la infección de la herida quirúrgica (3).

En todos los trabajos revisados por Sanz et al (4), en 2004, se comparó una formula inmunomoduladora frente a una dieta estándar y se administró en el postoperatorio inmediato. Observándose, en todos ellos, una reducción de las infecciones postoperatorias e incluso de la estancia hospitalaria.

En este estudio se evaluó el impacto de los diferentes tipos de suplementos nutricionales administrados a los pacientes ingresados en la Unidad de Hospitalización a Domicilio (UHD) con heridas quirúrgicas abdominales complicadas.

\section{MÉTODOS}

Estudio retrospectivo de los pacientes que a su ingreso en la Unidad de Hospitalización a Domicilio presentaban heridas quirúrgicas abdominales complicadas.

Se revisaron las historias clínicas y notas evolutivas de los pacientes ingresados con el grupo diagnóstico «quirúrgico» de los 5 años anteriores, seleccionando aquellas que presentaron los criterios de inclusión.

Criterios de inclusión: pacientes ingresados en la UHD que tuvieran como motivo de ingreso «herida quirúrgica abdominal complicada» y que se les hubiera administrado suplementación nutricional.

Variables explicativas: el tipo de suplemento recibido.

Variables resultado: albumina, tiempo de estancia hospitalaria y ciclos de antibioterapia recibidos durante su ingreso en la UHD.

Para la descripción de la población estudiada se utilizaron técnicas descriptivas; cálculo de frecuencias y, en el caso de las cuantitativas, medidas de tendencia central y desviación estándar (SD) o el intervalo intercuartílico (IIQ), cuando así lo aconsejó la distribución. Para el análisis entre variables, se utilizó los estadísticos Chi-cuadrado, t de student y ANOVA. 


\section{RESULTADOS}

Se revisaron 67 historias de pacientes que habían ingresado por herida quirúrgica abdominal complicada. La edad media fue de 69,2 $\pm 5,6$. El sexo predominante fue el femenino con 47 mujeres $(70,1 \%)$. Todos los pacientes presentaban heridas abdominales abiertas y el $36(53,7 \%)$ presentaban infección de la herida quirúrgica y estaban recibiendo antibioterapia intravenosa en el momento del ingreso. El Índice de Masa Corporal (IMC) medio fue de $26,43 \pm 4,59 \mathrm{~kg} / \mathrm{m} 2$, estando 47 de los pacientes $(70,1 \%)$ en cifras compatibles con el normopeso, $17(25,4 \%)$ se encontraban en el rango del sobrepeso u obesidad y $3(4,5 \%)$ en desnutrición calórica. Las cifras de albumina en 40 pacientes $(59,7 \%)$ se encontraron entre 3 y $3,5 \mathrm{~g} / \mathrm{dl}$ indicando una desnutrición proteica leve y en $11(16,4 \%)$ se situaban por debajo de los $3 \mathrm{~g} / \mathrm{dl}$. Todos los pacientes recibieron algún tipo de suplemento nutricional: 42 (62,7\%) pacientes una nutrición estándar, 17 (25,4\%) Inmunomodulador (Atempero®) y $8(11,9 \%)$ otros.

Al mes del ingreso, el valor medio de albumina se había incrementado en todos los pacientes (media al ingreso 2,46 vs media al mes 3,01 ; $p$-valor $=0,032$ ), aunque la mayor diferencia la presentaron los pacientes que recibieron suplemento inmunomodulador (media al mes 3,57; p-valor $=0,001)$. Los datos antropométricos no se encontraron recogidos en las notas evolutivas de 61 $(91,0 \%)$ de los pacientes; en los 6 pacientes que las reflejaron, un paciente había ganado $1 \mathrm{~kg}$ de peso y 5 habían perdido una media de peso de $740 \pm 251,0 \mathrm{~g}$, sin asociación con el tipo de suplementación recibida. El tiempo medio de ciclos de antibioterapia no presentó diferencias significativas ( $p$-valor $=0,897$ ), si bien 2 pacientes que estaban en el grupo de «otros» presentaron una segunda infección con necesidad de nuevos ciclos de antibioterapia. La estancia media fue de $84,05 \pm 17,48$ días hasta el alta, en el grupo del suplemento inmunomodulador fue de 77,18 \pm 13,72 días $(p$-valor $=0,001)$.

\section{DISCUSIÓN}

La cicatrización de las heridas es un proceso complejo, que depende en gran parte de los sustratos nutricionales disponibles. Cuando se usa junto con $\beta$-hidroxi $\beta$-metilbutirato, se ha demostrado que la arginina y la glutamina aumentan la deposición de colágeno en sujetos humanos (5). Sin embargo, no hay investigaciones experimentales sobre la influencia de mezcla de nutrientes consideradas Inmunomoduladoras con respecto a la cicatrización secundaria de heridas en humanos.

Desde que se introdujo la inmunonutrición como una alternativa para mejorar la evolución de los pacientes quirúrgicos ha habido muchos trabajos que han intentado demostrar que la suplementación perioperatoria con fórmulas Inmunomoduladoras pueden reducirla estancia hospitalaria y las complicaciones entre los pacientes sometidos a diversas cirugías electivas. Martín et al. (6), en 2017, publicaron un trabajo con 71 pacientes en el que determinaban que la administración preoperatoria de suplementos de inmunonutrición disminuía la estancia hospitalaria postoperatoria, las complicaciones infecciosas y la morbilidad en pacientes sometidos a cirugía de electroporación irreversible para el cáncer de páncreas localmente avanzado.

Stableforth et al. (7), en 2009, publicaron una revisión sistemática de 10 ensayos clínicos en pacientes con cirugía de cáncer de cabeza y cuello, y no encontraron reducciones en las complicaciones clínicas pero sí una asociación con la disminución de la estancia hospitalaria. Aunque expresaron que los ensayos fueron pequeños y con informes incompletos de los resultados.

Otra revisión sistemática con meta-análisis, publicada en 2018 por Cheng et al. (8), evaluaba el impacto de la inmunonutrición en pacientes con cáncer gástrico sometidos a una gastrectomía 
total, en índices bioquímicos, inmunes y resultados clínicos. Ellos incluyeron 7 estudios con 583 pacientes, encontrando mejoría en los parámetros de inmunidad celular e inflamatorios y reducción la función inmune para pacientes con cáncer gástrico después de la cirugía. Aunque la incidencia de infección pulmonar, la disminución de la estancia hospitalaria y otros resultados clínicos no mejoraron.

No son comparables los resultados de los trabajos localizados con nuestro trabajo. En el caso de este estudio, con pacientes que han sido sometidos a cirugía gastrointestinal, que se han complicado, presentando heridas abdominales abiertas y con procesos infecciosos, es el peor escenario posible para comprobar la efectividad de un suplemento nutricional y no hemos podido encontrar otro trabajo similar con quien poder contrastar nuestros resultados.

Por todo lo anteriormente señalado, se podría concluir: los datos apoyan un beneficio de la suplementación oral, en este tipo de pacientes, mejorando su estado nutricional. El grupo que se suplementó con Atempero®, destacó tanto en la recuperación de los niveles de albumina como en una mejor evolución, lo que se traduce en un alta significativamente más precoz.

\section{BIBLIOGRAFÍA}

1. Schloerb PR. Immune-enhacing diets: products, components, and their rationales. JPEN J Parenter Enteral Nutr. 2001;25(2 Suppl):S3-7. DOI: 10.1177 / 014860710102500202; PMID: 11288920

2. Faist E, Kupper TS, Baker CC, Chaudry IH, Dwyer J, Baue AE. Depression of cellular immunity after major injury. Its association with posttraumatic complications and its reversal with immunomodulation. Arch Surg. 1986; 121(9):1000-5. DOI: 10.1001/archsurg. 1986.01400090026004; PMID: 3741094

3. Moya P, Miranda E, Soriano-Irigaray L, Arroyo A, Aguilar MD, Bellón M, et al. Perioperative immunonutrition in normo-nourished patients undergoing laparoscopic colorectal resection. Surg Endosc. 2016;30(11):4946-53. DOI: 10.1007/s00464-016-4836-7; PMID: 26936601

4. Sanz A, Celaya S, Gracia P, Gracia MI, Albero R. Inmunonutrición. Endocrinol Nutr. 2004;51(4):202-17.

5. Wild T, Rahbarnia A, Kellner M, Sobotka L, Eberlein T. Basics in nutrition and wound healing. Nutrition. 2010;26(9):862-6. DOI: 10.1016/j.nut.2010.05.008; PMID: 20692599

6. Martin RC, Agle S, Schlegel M, Hayat T, Scoggins CR, McMasters KM, et al. Efficacy of preoperative immunonutrition in locally advanced pancreatic cancer undergoing irreversible electroporation (IRE). Eur J Surg Oncol. 2017; 43(4):772-9. DOI: 10.1016/j.ejso.2017.01.002; PMID: 28162818

7. Stableforth WD, Thomas S, Lewis SJ. A systematic review of the role of immunonutrition in patients undergoing surgery for head and neck cancer. Int J Oral Maxillofac Surg. 2009;38(2):10310. DOI: 10.1016/j.ijom.2008.12.008; PMID: 19144498

8. Cheng $Y$, Zhang J, Zhang L, Wu J, Zhan Z. Enteral immunonutrition versus enteral nutrition for gastric cancer patients undergoing a total gastrectomy: a systematic review and meta-analysis. BMC Gastroenterol. 2018;18(1):11. DOI: 10.1186/s12876-018-0741-y; PMID: 29338698 